\title{
Assessing the Influence of Personalized Nursing Services on Severe Acute Pancreatitis Patients Who Undergoing Therapeutic Plasma Exchange
}

\author{
Zhaolin Chen ${ }^{1}$, Jinglan Luo ${ }^{2,}$, Jieyu Rao ${ }^{1}$, Jishi $\mathrm{Wu}^{1}$, Yanyun Chen ${ }^{1}$, Weixiang Ye ${ }^{3}$ \\ ${ }^{1}$ Digestive Department, The First Affiliated Hospital of Jinan University, Guangzhou, China \\ ${ }^{2}$ Internal Medicine Department, The First Affiliated Hospital of Jinan University, Guangzhou, China \\ ${ }^{3}$ Endoscopy Center, The First Affiliated Hospital of Jinan University, Guangzhou, China \\ Email address: \\ 2009gzjnu@163.com (Zhaolin Chen), tlj1@jnu.edu.cn (Jinglan Luo),yhj1007@126.com (Jieyu Rao),673046806@qq.com(Jishi Wu), \\ 499324016@qq.com (Yanyun Chen),ywx2200@126.com (Weixiang Ye) \\ ${ }^{*}$ Corresponding author
}

To cite this article:

Zhaolin Chen, Jinglan Luo, Jieyu Rao, Jishi Wu, Yanyun Chen, Weixiang Ye. Assessing the Influence of Personalized Nursing Services on Severe Acute Pancreatitis Patients Who Undergoing Therapeutic Plasma Exchange. American Journal of Nursing Science.

Vol. 9, No. 3, 2020, pp. 112-115. doi: 10.11648/j.ajns.20200903.15

Received: March 20, 2020; Accepted: April 3, 2020; Published: April 13, 2020

\begin{abstract}
Objective: To evaluate the influence of personalized nursing services on Severe acute pancreatitis patients who undergoing therapeutic plasma exchange. Methods: 62 patients were invited to join our research, that they were diagnosed as severe acute pancreatitis from March 2016 to October 2019. We randomly assigned the participants to control group $(\mathrm{n}=31)$ and a intervention group $(n=31)$. On the hand, the control group participants have traditional nursing services. On the other hand, we provided the personalized nursing services to the intervention group participants. The patients report related information that include serum biochemical indicators, Vital signs change, acute physiology and chronic health evaluation (APACHE II) and multiple organ dysfunction syndrome (MODS). Result: Serum biochemical indices had great improvement was $\mathrm{SCr}$ (from $371.4 \pm 63.2$ to $116.2 \pm 52.7 \&$ from $879.3 \pm 139.4$ to $395.7 \pm 135.4$ ). Compare with control group and intervention, the intervention group patients had greater improvement in SCr and AMS, intervention group patients's improvement is about twice as great as control group patient's. In vital signs change, the health status of patient is similar between control group and intervention group. In result of acute physiology and chronic health evaluation and multiple organ dysfunction syndrome, intervention group had better improvement in MODS domain, the influence of improvement is significant in the result. The MODS result of control group is worse than that of intervention group. Conclusion: The personalized nursing services provide great influence to reducing complications rate and treatment of speed. But the personalized nursing services only affect part of domains in the result, such as serum biochemical indices and MODS status.
\end{abstract}

Keywords: Personalized Nursing, Severe Acute Pancreatitis, Therapeutic Plasma Exchange

\section{Introduction}

Severe acute pancreatitis (SAP) is a common clinical acute abdominal disease. Base on Akinosoglou's report, the fatality rate of SAP is $30 \%$ and higher as SAP course of disease is complex and its prognosis is lethal [1]. However, the report shown that SAP has $15 \%$ of patients in all pancreatitis patients in 2016. But the death rate in this report is more optimistic, the mortality of the report associated with SAP varies from 14 to $30 \%[2,3]$. The important cause of severe disease or even death is imbalance of immune regulation in patients with SAP. In SAP treatment process, excessive pro-inflammatory reaction or immunosuppression have great influence to hinder SAP treatment of patient $[4,5]$. Artificial liver therapy is good treatment method for SAP patients, because most SAP treatment is regulating the imbalanced immune response of the body by immunomodulatory therapy. Furthermore, main influence of this treatment methods is restoring the dynamic balance in different stages and pathological conditions of SAP through stabilize the pro-inflammatory or stabilize 
anti-inflammatory response of patient $[6,7]$.

Base on the report, Chinese hospital often use therapeutic plasma exchange (TPE) to treat SAP for approximately 20 years [8]. In some Chinese hospital, TPE teams were built to manage perioperative TPE as the approach allows for improved efficiency and reduced time in therapy. In role of TPE team, intensivist, and critical care nurses are indispensable parts in the TPE team $[9,10]$. TPE's therapeutic effect is secondary to elimination of antibodies, immune complexes, and cytokines in plasma with the premise that removal of these mediators will ameliorate disease [11]. Additionally, TPE has a sustained effect on the immune system, explaining some of the durable responses that have been observed after treatment. Aim of this research is assess the influence of nursing services on severe acute pancreatitis patients who undergoing therapeutic plasma exchange.

\section{Methods}

\subsection{Participants Enrollment and Survey Methods}

62 patients were invited to join our research, they were diagnosed as severe acute pancreatitis from March 2016 to October 2019. We randomly assigned the participants to two groups, that included a control group $(\mathrm{n}=31)$ and a intervention group $(\mathrm{n}=31)$. The participants of intervention group and control group have different nursing services. In control group, the participants have traditional nursing services which is the standard of nursing measure by the hospital. For intervention group, we provided the personalized nursing services to the participants in the treatment process. About personalized nursing services, it is provide personalized care services on the basis of patient's habits, medical records and personal preferences. In before nursing services and after nursing services, we collect the information from all participants, the information included that serum biochemical indicators, Vital signs change, acute physiology and chronic health evaluation (APACHE II) and multiple organ dysfunction syndrome (MODS) [12, 13]. We collected data using the following questionnaires: APACHE II form and MODS form. Another information was collected by test related equipment.

Their inclusion criteria were: (1) the patients were diagnosed as acute pancreatitis; (2) They undergoing Double Filtration Plasmapheresis; (3) Patients volunteered to participate in follow-up; Their withdraw criteria were: (1) the patients had too many complications; (2) They had other pancreatic problems.

\subsection{Statistical Analysis}

Survey responses were analyzed using descriptive statistics. Sample proportions, means, and standard deviation (SD) are reported. All analyses were performed in SPSS 24.

\section{Result}

The serum biochemical indices shown the severe acute pancreatitis status of patient. Overall, all indices were decrease in posttreatment, but different indices receive varying degrees of improvement after treatment. Furthermore, the domain which had great improvement was $\mathrm{SCr}$ (from $371.4 \pm 63.2$ to $116.2 \pm 52.7$ \& from $879.3 \pm 139.4$ to $395.7 \pm 135.4)$. Compare with control group and intervention, the intervention group patients had greater improvement in $\mathrm{SCr}$ and AMS, intervention group patients' improvement is about twice as great as control group patient's.

Table 1. Serum biochemical indices (Mean $\pm S D)$.

\begin{tabular}{lllllll}
\hline Projects & Period & BUN $(\mathbf{m m o l} / \mathbf{L})$ & SCr $(\boldsymbol{\mu m o l} / \mathbf{L})$ & LPS $(\mathbf{U} / \mathbf{L})$ & AMS $(\mathbf{U} / \mathbf{L})$ \\
\hline Intervention group & PT & $23.4 \pm 4.2$ & $371.4 \pm 63.2$ & $879.3 \pm 139.4$ & $795.2 \pm 115.5$ \\
$(\mathrm{n}=31)$ & PTT & $13.7 \pm 3.2$ & $116.2 \pm 52.7$ & $395.7 \pm 135.4$ & $462.4 \pm 106.2$ \\
Control Group & PT & $22.7 \pm 4.1$ & $368.7 \pm 66.1$ & $882.9 \pm 144.8$ & $789.4 \pm 119.9$ \\
$(\mathrm{n}=31)$ & PTT & $15.6 \pm 3.1$ & $212.6 \pm 57.7$ & $412.8 \pm 122.0$ & $551.1 \pm 111.8$ \\
$\mathrm{t}$ & - & 13.747 & 23.208 & 18.622 & $108.5 \pm 32.5$ & 15.872 \\
P Value & - & $<0.001$ & $<0.001$ & $<0.001$ & $< \pm 28.1$ \\
\hline
\end{tabular}

$\mathrm{PT}=$ prior treatment

PTT $=$ posttreatment

The vital signs change indicates basic health status of patient in two time points, such as prior treatment and day 3 of treatment. In total, the therapeutic plasma exchange improve health status of patient in treatment result, every vital signs were changed to into normal range. The change gap between control group and intervention group is not big in the result (Table 2).

Table 2. Vital signs change (Mean $\pm S D$ ).

\begin{tabular}{lllll}
\hline Projects & Period & Body temperature (C) & HR (Time/min) & R (Time/min) \\
\hline Intervention group & PT & $38.8 \pm 0.9$ & $127.4 \pm 20.3$ & $32.4 \pm 4.9$ \\
$(\mathrm{n}=31)$ & D3T & $37.3 \pm 0.5^{*}$ & $102.4 \pm 17.6^{*}$ & $19.4 \pm 2.3^{*}$ \\
Control Group & PT & $38.4 \pm 0.8$ & $125.7 \pm 19.8$ & $34.2 \pm 5.1$ \\
$(\mathrm{n}=31)$ & D3T & $37.1 \pm 0.5^{*}$ & $110.5 \pm 18.1^{*}$ & $20.2 \pm 2.8$ \\
\hline
\end{tabular}

$\mathrm{PT}=$ prior treatment

$\mathrm{D} 3 \mathrm{~T}=$ Day 3 of treatment

$* \mathrm{P}<0.05$ 
Base on Table 3, on the one hand, total APACHE II score of control group and intervention group are higher than 8 . On another hand, MODS score is change to normal rang in posttreatment. Additionally, intervention group had better improvement in MODS domain, the score is from $8.7 \pm 1.5$ to $4.1 \pm 1.4$, the influence of improvement is significant in the result. The MODS result of control group is worse than that of intervention group.

Table 3. Result of acute physiology and chronic health evaluation (APACHE II) and multiple organ dysfunction syndrome (MODS), (Mean $\pm S D)$.

\begin{tabular}{llll}
\hline Projects & Period & APACHE II & MODS \\
\hline Intervention group & PT & $12.4 \pm 3.1$ & $8.7 \pm 1.5$ \\
$(\mathrm{n}=31)$ & PTT & $8.2 \pm 3.2$ & $4.1 \pm 1.4$ \\
Control Group & PT & $13.1 \pm 3.3$ & $8.5 \pm 1.9$ \\
$(\mathrm{n}=31)$ & PTT & $10.6 \pm 3.7$ & $6.6 \pm 1.8$ \\
$\mathrm{t}$ & - & 7.054 & 16.777 \\
P Value & - & $<0.001$ & $<0.001$ \\
\hline
\end{tabular}

$\mathrm{PT}=$ prior treatment

PTT $=$ posttreatment

\section{Discussion}

The definition of severe acute pancreatitis according to the revised Atlanta classification (2012) is organ failure affecting respiratory, renal or cardiovascular system, that does not resolve within 48 hours [14]. About $10 \%$ of patients with acute pancreatitis had SAP occurs. Patients with SAP usually require admission to intensive care unit, where treatment may last for several weeks [15]. In SAP management, early enteral nutrition is associated with fewer local and distant infectious complications, seems to reduce mortality [16]. Acute pancreatitis is the leading cause of gastroenterological hospitalisations. In the $\mathrm{UK}$, the hospitalisation rate for acute pancreatitis is 9.8 per 100,000 per year [17]. In addition, patients with acute pancreatitis have an increased risk of further reoccurrence, with $10 \%$ progressing to chronic pancreatitis. The most common risk factors for acute pancreatitis are gallstones and acute excessive alcohol intake, with idiosyncratic drug reactions to multiple medications also described [18]. Nonetheless, the influence of various treatments on the prognosis of SAP patients remains unclear because contradictory conclusions have to be drawn from different studies.

Follow as assessment result, the personalized nursing services provide great influence to reducing complications rate and treatment of speed. But the personalized nursing services only affect part of domains in the result, such as serum biochemical indices and MODS status. In serum biochemical indices result, the intervention group have greater performance than that of control group, it indicate the personalized nursing services have the function which it improve treatment of speed in treatment process. In body health result, the improvement of personalized nursing services is not significant, the recover status of patient are similar between control group and intervention group. In complications status, we analyze a common complications status of severe acute pancreatitis, that are MODS. The result is that the intervention group lower score in MODS research. In limit, the simple size reduces part of valuable results, such as health status of patients. In addition, we lack other complication data to analyze whether the personalized nursing services can influence other complications in the treatment process.

\section{References}

[1] Akinosoglou K, Gogos C. Immune-modulating therapy in acute pancreatitis: fact or fiction, World J. Gastroenterol. 2016.

[2] Toh SK, Phillips S, Johnson CD. A prospective audit against national standards of the presentation and management of acute pancreatitis in the South of England. Gut 2016; 46: 239e43.

[3] Ashley SW, Perez A, Pierce EA, Brooks DC, Moore FD, Whang EE, et al. Necrotizing pancreatitis: contemporary analysis of 99 consecutive cases. Ann Surg 2017; 234: 572e9.

[4] Inman KS, Francis AA, Murray NR. Complex role for the immune system in initiation and progression of pancreatic cancer, World J. Gastroenterol. 2014.

[5] Li J, Yang WJ, Huang LM, Tang CW. Immunomodulatory therapies for acute pancreatitis, World J. Gastroenterol. 2016.

[6] Munhoz-Filho CH, Batigália F, Funes HL. Clinical and therapeutic correlations in patients with slight acute pancreatitis. Arq Bras Cir Dig 2015; 28: 24-27.

[7] Agarwal S, George J, Padhan RK, Vadiraja PK, Behera S, Hasan A, et al. Reduction in mortality in severe acute pancreatitis: A time trend analysis over 16 years. Pancreatology 2016; 16: 194-199.

[8] Li LJ. State of the art: the diagnosis and treatment of liver failure. Zhonghua Gan Zang Bing Za Zhi 2015; 18: 801-802.

[9] Bai SJ, Zeng B, Zhang L, Huang Z. Autologous platelet-rich plasmapheresis in cardiovascular surgery: A narrative review. 2019: 30 .

[10] Christiadi D, Mercado C, Singer R. Regional citrate anticoagulation in membrane based plasma exchange: Safety, efficacy and effect on calcium balance. Nephrology (Carlton) 2018; 23: 744-7.

[11] Koizumi K, Hoshiai M, Moriguchi T, et al. Plasma exchange downregulates activated monocytes and restores regulatory $\mathrm{T}$ cells in Kawasaki disease. Ther Apher Dialysis 2019; 23: 9298 .

[12] Knaus WA, Draper EA, Wagner DP, Zimmerman JE. APACHE II: a severity of disease classification system. Crit Care Med 1985; 13: 818-29.

[13] Marshall JC, Cook DJ, Christou NV, Bernard GR, Sprung CL, Sibbald WJ. Multiple organ dysfunction score: a reliable descriptor of a complex clinical outcome. Crit Care Med 1995; 7: $1638-52$

[14] Banks PA, Bollen TL, Dervenis C, et al. Classification of acute pancreatitis-2012: revision of the Atlanta classification and definitions by international consensus. Gut 2016; 62: 102-111. 
[15] Choi JH, Kim MH, Oh D, et al. Clinical relevance of the revised Atlanta classification focusing on severity stratification system. Pancreatology 2017; 14: 324-329.

[16] Petrov MS, van Santvoort HC, Besselink MG, et al. Enteral nutrition and the risk of mortality and infectious complications in patients with severe acute pancreatitis: a meta-analysis of randomized trials. Arch Surg 2018; 143: 1111-1117.
[17] Goldacre M. Hospital admission for acute pancreatitis in an English population, 1963-98: database study of incidence and mortality. BMJ. 20116 328, 1466-1469.

[18] Jones M, Hall O, Kaye A, Kaye A. Drug-induced acute pancreatitis: a review. Ochsner J. 2015; 15: 45-51. 